Tropical Journal of Pharmaceutical Research, August 2009; 8 (4): 303-310

(C) Pharmacotherapy Group,

Faculty of Pharmacy, University of Benin

Benin City, 300001 Nigeria.

All rights reserved.

Research Article

Available online at http://www.tjpr.org

\title{
Clinical Features, Complications and Treatment Outcome of Brucella infection: Ten Years' Experience in an Endemic Area
}

\section{HA Bukharie}

Department of Internal Medicine, Division of Infectious Disease, King Fahd Hospital of the University, Alkhobar, Saudi Arabia

\begin{abstract}
Purpose: Brucellosis is a multi-systemic infection that is endemic in some parts of the world. The purpose of this study was to examine the epidemiology as well as the clinical and haematological characteristics, complications, and treatment outcome of patients with brucellosis at the King Fahd Hospital of the University Alkhobar, Saudi Arabia.

Methods: A descriptive case assessment of all adult patients (aged 13 years and above) with brucellosis over a period of 10 years between 1998 and 2007 was undertaken.

Results: The diagnosis of brucellosis in 84 patients was made by clinical findings, positive agglutination titre, and/or the isolation of Brucella species. The number of patients with brucellosis presenting at our hospital reduced over the study period, with a much smaller proportion of cases occurring after the year 2000. Most of the patients had fever (73\%) and musculoskeletal symptoms (64\%). Splenomegaly was recorded in $13 \%$ and hepatomegaly in $11 \%$ of patients. Seven patients (8\%) had epididymo-orchitis. Two patients presented with fever of unknown origin with negative serology and only bone marrow culture was positive for Brucella mellitensis. Two patients presented with menstrual associated recurrent fever. One previously healthy patient presented with jaundice and spontaneous peritonitis. No therapeutic failure was observed but relapse occurred in six patients (7\%). None of the patients who received streptomycin or ciprofloxacin as part of their antibiotic regimen had a relapse.

Conclusion: Brucellosis has a wide range of clinical manifestations. Although the number of patients with brucellosis presenting at our hospital has reduced, continuous organized effort is necessary for brucellosis control.
\end{abstract}

Key words: Brucellosis; Recurrent fever; Saudi Arabia; Ascites; Arthritis; Menstrual cycle. 


\section{INTRODUCTION}

Brucellosis is a disease of domestic and wild animals that can be transmitted to humans (zoonosis). The disease exists worldwide, particularly in the Mediterranean basin, the Arabian Peninsula, the Indian subcontinent, and in parts of Mexico and Central and South America ${ }^{1}$. Consumption of contaminated foods and occupational contact remain the main sources of infection ${ }^{1}$. Human brucellosis may present with protean clinical manifestations that require broad differential diagnosis, including many infectious and noninfectious diseases. It is a systemic infection that can affect any organ in the body ${ }^{1}$. The onset of disease is insidious in approximately half of all cases. It is characterized by several somatic complaints such as fever, sweats, anorexia, and weight loss. By contrast, there can be few abnormal physical findings. Occasionally, symptoms related to a single organ predominate. The aim of this study was to examine the epidemiological features, clinical and haematological characteristics, complications, and treatment outcomes of brucellosis in an endemic region.

\section{PATIENTS AND METHODS}

\section{Study population}

Between 1998 and 2007, a descriptive case assessment of 84 adult patients with brucellosis presented to King Fahd Hospital of the University, Alkhobar, in a longitudinal prospective study. The university hospital is a secondary-tertiary care hospital in the Eastern Province of Saudi Arabia.

\section{Diagnostic criteria}

The diagnosis of brucellosis was based on the clinical findings characteristic of the disease and on either positive cultures that tested for Brucella species or a titre of $\geq 1$ : 160 antibodies by tube agglutination. Complete blood count, sedimentation rate, liver function tests, and in some patients detection of immunoglobulin (IgM and $\lg G$ ) antibodies by ELISA were also performed.

\section{Treatment and outcome}

Various treatment combinations of antiBrucella antimicrobials (streptomycin, doxycycline, co-trimoxazole, rifampicin, ciprofloxacin) were used, depending on the patient's age and pregnancy status. The mean follow-up period was one year, although this was not possible for all patients because of the nomadic nature of the patient population. Relapse was defined as an initial improvement followed by reappearance of symptoms and signs during the treatment period or within 2 months of discontinuation of treatment. A cure was defined as no recurrence of symptoms and signs during the 12-month follow-up period.

\section{RESULTS}

A total of 84 patients were diagnosed with brucellosis during the 10-year study. The number of patients with brucellosis reduced during the study period, with a much smaller number of cases occurring after 2000 (Fig. 1).

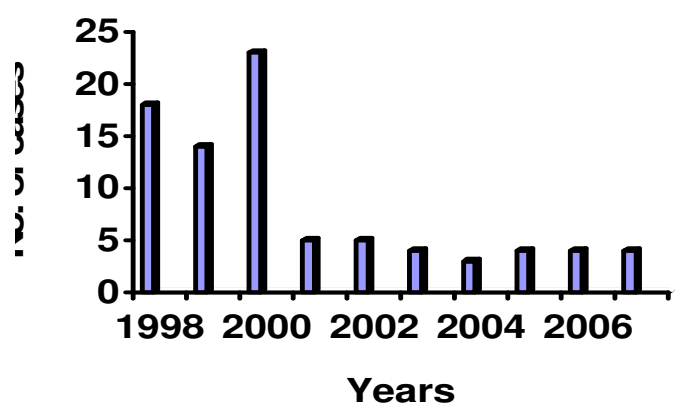

Fig.1: The distribution of Brucella cases between 1998 and 2007

The median age of patients was $32 \pm 9$ years (range: 13-81 years). Males were more often affected than females and the male-to-female ratio was $3: 1$. Of the 84 patients, two presented with a relapse, while the other 82 
were diagnosed with brucellosis for the first time. Fifty four patients (64\%) gave history of contact with animals, sixteen patients (19\%) gave history of drinking raw milk, five patients $(6 \%)$ were involved in slaughtering animals particularly during Hajj season, three patients $(4 \%)$ were laboratory workers, and six patients $(7 \%)$ denied any risk factors for acquiring brucellosis. The occupations of the patients varied from farmers (21 males and 2 females), Sheppard (12 males, and 1 female), students (8 males and 4 females), teachers (6 males and 3 female), laboratory workers (2 males, and 1 female), engineer (I male), housemaid (1 female), housewives (18 females), and unemployed (4 males).

The clinical characteristics of the 84 patients with brucellosis are described in Table 1. A history of fever was obtained from $95 \%$ of patients, but it was positive in $73 \%$ of patients. A substantial number of patients (64\%) developed arthritis or arthralgia. Splenomegaly was recorded in $13(16 \%)$ and hepatomegaly in 9 (11\%) patients. Two patients presented with fever of unknown origin with negative serology and only bone marrow culture was positive for Brucella mellitensis. Two patients presented with recurrent fever associated with menstruation.

Table 1: Clinical characteristics and laboratory findings of 84 patients with brucellosis

\begin{tabular}{|c|c|c|c|}
\hline Symptom/sign & $\begin{array}{l}\text { Number of } \\
\text { patients (\%) }\end{array}$ & Laboratory findings & $\begin{array}{l}\text { Number of } \\
\text { patients (\%) }\end{array}$ \\
\hline $\begin{array}{l}T_{\max }(\text { axillary) } \\
\geq 39^{\circ} \mathrm{C} \\
\geq 37.5^{\circ} \mathrm{C}<39{ }^{\circ} \mathrm{C} \\
<37.5^{\circ} \mathrm{C} \text { (afebrile) } \\
\text { Recurrent fever }\end{array}$ & $\begin{array}{c}61(73) \\
39(46) \\
22(26) \\
23(28) \\
2(2)\end{array}$ & $\begin{array}{l}\text { Anemia } \\
\text { (hemoglobin concentration }<11.5 \mathrm{~g} / \mathrm{dL} \\
\text { in females and }<13.5 \mathrm{~g} / \mathrm{dL} \text { in males) }\end{array}$ & $30(36)$ \\
\hline Arhthritis/arthralgia & $54(64)$ & $\begin{array}{l}\text { Thrombocytopenia } \\
\left(<150,000 \mathrm{~mm}^{-3}\right)\end{array}$ & $3(4)$ \\
\hline Hepatomegaly & $9(11)$ & Leukopenia $\left(<4500 \mathrm{~mm}^{-3}\right)$ & $5(6)$ \\
\hline Lymphadenitis & $11(13)$ & Leukocytosis $\left(>11,000 \mathrm{~mm}^{-3}\right)$ & $5(6)$ \\
\hline Splenomegaly & $13(16)$ & Lymphocytosis (>2500 $\mathrm{mm}^{-3}$ ) & $50(60)$ \\
\hline Fever of unknown origin & $2(2)$ & Pancytopenia & $1(1)$ \\
\hline Epididymo-orchitis & $7(8)$ & $\operatorname{ESR}(\mathrm{mm} / \mathrm{h})$, median & 35 \\
\hline Endocarditis & $1(1)$ & Increased AST and/or ALT & $24(29)$ \\
\hline Abortion & $1(1)$ & $\begin{array}{l}\text { Increased GGT and alkaline } \\
\text { phosphatase }\end{array}$ & $31(37)$ \\
\hline Neurobrucellosis & $1(1)$ & SAT(titer, $\geq 1: 160$ ) & $80(95)$ \\
\hline Cough & $1(1)$ & $\begin{array}{l}\text { ELISA for IgM and IgG(done in } 28 \\
\text { patients) }\end{array}$ & $18(64)$ \\
\hline Abdominal pain & $1(1)$ & Positive culture for Brucella species & $31(37)$ \\
\hline Ascites & $1(1)$ & Blood cultures & $2(2)$ \\
\hline Jaundice & $1(1)$ & Bone marrow aspirates & \\
\hline
\end{tabular}

$S A T$ - standard tube agglutination test, $A L T$ - alanine aminotransferase, AST - aspartate aminotransferase 
One previously healthy patient presented with jaundice and spontaneous peritonitis. The diagnosis was confirmed after isolating Brucella melitensis from blood and by complete resolution of symptoms with treatment. Eleven patients (13\%) presented with generalized lymphadenopathy. Seven patients $(8 \%)$ had epididymo-orchitis. Neurological and cardiac involvement was infrequent. One patient had meningitis and another patient presented with endocarditis.

\section{Laboratory data}

Erythrocyte sedimentation rate (ESR) was measured in all patients and it ranged from 4 to $110 \mathrm{~mm} /$ hour (median $35 \mathrm{~mm} /$ hour). Thirty patients $(36 \%)$ had anaemia (haemoglobin concentration $<11.5 \mathrm{~g} / \mathrm{dL}$ in females and $<13.5$ $\mathrm{g} / \mathrm{dL}$ in males). Five patients had leukocytosis $\left(>10,500\right.$ white blood cells [WBCs] $\left./ \mathrm{mm}^{3}\right)$, and five patients had leukopaenia $(<4500$ WBCs $/ \mathrm{mm}^{3}$ ). Lymphocytosis was seen in 50 patients $(60 \%)$. Three patients $(4 \%)$ had thrombocytopenia $\left(<150,000\right.$ platelets $\left./ \mathrm{mm}^{3}\right)$. Twenty-four (29\%) patients had slight-tomoderately increased serum hepatic transaminase levels. Thirty-one patients (37\%) had increased serum $\gamma$-glutamyl transpeptidase (GGT) and alkaline phosphatase concentrations with normal bilirubin levels (dissociated cholestasis).

Standard tube agglutination testing of initial samples from $80(95 \%)$ of the 84 patients was positive for antibodies to Brucella (titre, $\geq$ 1: 160). Cultures of specimens from 33 (39\%) of the 84 patients were positive for Brucella species, thirty-one were isolated from blood samples, and two from bone marrow aspirates. Only 18 of the 33 Brucella isolates were speciated; this was because some isolates died, some specimens were discarded, or laboratory reagents were not available. Of these 18 isolates, twelve (67\%) were B. melitensis and six (33\%) were Brucella abortus. Antimicrobial susceptibility testing was carried out on 33 isolates. The number of Brucella isolates with antimicrobial resistance was as follows: $12(36 \%)$ were resistant to co-trimoxazole; 6 (18\%) were resistant to rifampicin; all isolates were susceptible to tetracycline, streptomycin, and ciprofloxacin.

ELISA was performed to test for $\lg \mathrm{M}$ and $\lg \mathrm{G}$ antibodies in 28 patients, and only 18 (64\%) were positive for IgM or IgG antibodies. A positive result was noted in 8 of 23 patients (34\%) for culture-confirmed brucellosis.

\section{Treatment and rate of relapse}

Treatment regimens for the 84 patients consisted of combinations of two or three agents: streptomycin plus doxycycline (18\%); doxycycline plus rifampicin (18\%); doxycycline plus co-trimoxazole (12\%); rifampicin plus cotrimoxazole (2\%); doxycycline plus ciprofloxacin (31\%); and streptomycin plus doxycycline plus ciprofloxacin (19\%). Streptomycin was administered for 15 days. Treatment with other antimicrobial agents varied according to clinical response and the development of complications (mainly arthritis). Spondylitis is recognized as the most common and one of the most debilitating forms of focal brucellosis and there is no consensus on the best therapeutic regimens, so we chose triple therapy (doxycycline, streptomycin, and ciprofloxacin) to treat patients with Brucella spondylitis.

A total of 61 patients (73\%) had long-term follow-up (12 months). Six patients (7\%) suffered a relapse after completion of therapy with no osteoarticular or nervous system involvement. Three of these six patients had received doxycycline and co-trimoxazole. Isolates from two patients were found to be resistant to co-trimoxazole and the isolate from the third patient failed to grow. Three patients relapsed after receiving doxycycline and rifampicin. Blood cultures of both of these patients tested negative for Brucella. None of the patients who received streptomycin or ciprofloxacin in combination with doxycycline suffered a relapse (Table 2 ). 
Table 2: Treatment and outcome for 84 patients with brucellosis

\begin{tabular}{llll}
\hline Treatment & Patients $(\%)$ & Relapse rate $(\%)$ & Cure rate $(\%)$ \\
\hline Dox + Rif & $15(18)$ & $3(13)$ & $13(87)$ \\
Dox + Stm & $15(18)$ & 0 & $15(100)$ \\
Dox + Cipro & $26(31)$ & 0 & $26(100)$ \\
Dox + TMP-SMZ & $10(12)$ & $3(30)$ & $7(70)$ \\
TMP-SMZ+ Rif & $2(2)$ & 0 & $2(100)$ \\
Dox + Stm+ Cipro & $16(19)$ & 0 & $16(100)$ \\
Total & $84(100)$ & $6(7)$ & $78(93)$ \\
\hline
\end{tabular}

Note: The proportion of patients (\%) receiving each regimen are shown; Dox - doxycycline; Rif - rifampin; Stm - streptomycin; TMP-SMZ - trimethoprim-sulfamethoxazole; cipro - ciprofloxacin

\section{DISCUSSION}

Brucella is one of the world's main zoonotic pathogens, and is responsible for enormous economic losses, as well as considerable human morbidity in endemic areas. Brucellosis has been present for thousands of years and has managed to elude eradication ${ }^{2}$ even in the most developed countries ${ }^{2-4}$. It is well known to have a high prevalence in certain geographic areas, such as the Mediterranean basin, the Arabian Peninsula, the Indian subcontinent, and South America ${ }^{1-4}$.

Transmission of brucellosis to humans occurs through the consumption of infected, unpasteurized animal-milk products, by direct contact with infected animal parts (such as the placenta by inoculation through ruptures of skin and mucous membranes), and through the inhalation of infected aerosolized particles. Brucellosis is an occupational disease for shepherds, abattoir workers, veterinarians, dairy-industry professionals, and personnel in microbiologic laboratories.

The number of patients with brucellosis in our hospital reduced over the 10-year study period, with a much smaller proportion of cases occurring after 2000 . This is due to the concerted efforts of public health measures such as public education, milk pasteurization, and livestock immunization. In addition, increased awareness of the disease among physicians, improved laboratory diagnosis, and treatment of cases by primary care physicians may also have contributed to this reduction ${ }^{5}$.

Human brucellosis is traditionally described as a disease of protean manifestations, and the demographic and clinical characteristics of the patients in this study emphasize the wide and unexpected spectrum of this disease. The characteristics of fever vary and can be spiking and accompanied by rigors, or may be relapsing, mild, or protracted. Constitutional symptoms are generally present. Dissemination via the bloodstream can result in involvement of almost any organ and the clinical presentation can be misleading and cases in which gastrointestinal, respiratory, dermal, or neurological manifestations predominate are not uncommon ${ }^{6-10}$. However neurological or cardiovascular manifestation were rare in our patients. The results of physical examinations are often nonspecific, although lymphadenopathy, hepatomegaly, or splenomegaly is often present. Hepatomegaly was observed in $9 \%$ of cases, whereas splenomegaly and lymphadenopathy were present in $13 \%$ of cases.

Osteoarticular disease is the most common complication of brucellosis, and was reported in $64 \%$ of patients in our study. There are three distinct forms: peripheral arthritis, sacroilitis, and spondylitis. The reproductive system is the second most common site of focal brucellosis. Brucellosis can present as epididymo-orchitis in men and is often difficult to differentiate from other local disease ${ }^{11}$. Brucellosis during pregnancy is associated 
with a substantial risk of spontaneous abortion $^{12}$. We diagnosed epididymo-orchitis in seven patients, and one patient had an abortion.

Hepatitis is common, usually manifesting as mild transaminasemia ${ }^{13,14}$. In our study $29 \%$ of patients had increased enzyme concentrations (ALT, AST). Jaundice is usually rare and only one of the patients in our study presented with jaundice, together with spontaneous peritonitis. The patient had no underlying condition such as chronic liver disease, congestive heart failure, nor was she on peritoneal dialysis. Brucellosis is a rare cause of bacterial peritonitis. Few cases have been reported, and most were associated with chronic liver disease ${ }^{15}$. The other patients had underlying conditions such as ascites caused by congestive heart failure ${ }^{15}$ and in patients undergoing $C A P D^{16}$. Our patient had complete resolution of symptoms with treatment. Two patients presented with menstrual associated recurrent fever. Although brucellosis is known to cause recurrent fever, the association with menstrual cycle to my knowledge has never been reported.

Abnormal blood test results are usually mild and nonspecific. Changes in blood count are often due to mild leukopaenia and relative lymphocytosis, together with mild anaemia and thrombocytopaenia. The most common haematological manifestations in our study were anaemia, lymphocytosis, and moderate increases in the sedimentation rate. In our study, one patient presented with pancytopenia. Pancytopaenia in brucellosis is multifactorial and has been attributed to hypersplenism and bone marrow involvement.

The absolute diagnosis of brucellosis requires isolation of the bacterium from blood or tissue samples, or the combination of clinical symptoms and positive serology. The sensitivity of blood culture varies, depending on individual laboratory practices and how the cultures are obtained. Positive blood cultures are found in 15 to $70 \%$ of patients with brucellosis $^{17}$. In our study, positive cultures were obtained from $37 \%$ of blood and $2 \%$ of bone marrow samples of patients with brucellosis. Prior use of antibiotics is associated with reduced incidence of positive blood cultures but does not affect bone marrow culture. In two cases, patients had positive bone marrow cultures despite all other negative diagnostic tests for Brucella. A bone marrow culture is recommended for patients with fever of unknown origin, negative serology, and unexplained joint or hematological involvement in whom brucellosis is suspected $^{18}$.

The presumptive diagnosis of brucellosis can be made serologically in conjunction with compatible clinical presentation ${ }^{19}$. Positive results (titers of antibodies to Brucella of $\geq$ 1: 160 [standard tube agglutination test] or $\geq$ 1: 320 [Coombs' test]) are common, although low titers, determined by standard tube agglutination tests, have been reported ${ }^{18}$. In rare cases patients with brucellosis can have positive blood cultures but negative serology ${ }^{20}$. Two of the patients in our study with Brucella bacteremia had negative serology on repeated occasions. More recently, the Brucella enzyme-linked immunosorbent assay (ELISA) test has been introduced into clinical laboratories for the diagnosis of brucellosis. The ELISA test is reported to be rapid, highly sensitive, and specific for detecting the Brucella-specific lgG, $\lg \mathrm{M}$, and $\lg \mathrm{A}$ antibodies in blood and cerebrospinal fluid ${ }^{21}$. In our study, in patients with Brucella bacteremia the sensitivity of the ELISA IgM or IgG tests were lower than the sensitivity of the standard tube agglutination tests; this finding has also been reported by others $^{22}$.

The successful treatment of brucellosis requires prolonged chemotherapy regimen with a combination of antibiotics. In 1986, the World Health Organization issued guidelines for the treatment of human brucellosis ${ }^{23}$. The guidelines recommend two regimens, both involved doxycycline for six weeks and in combination with either streptomycin for two to three weeks or rifampin for six weeks. Both 
combination regimens are the most popular treatments worldwide, although they are not used universally. The streptomycin-containing regimen is slightly more effective in preventing relapse $^{24}$. However, parenteral administration of streptomycin requires either hospital admission or an adequate health care network - both of which are often absent from areas of endemic disease. On the other hand, the use of rifampin in areas in which brucellosis is endemic, and where tuberculosis is also usually endemic, raises concerns about the development of community resistance to rifampin. Al -Hajjaj et al documented worsening resistance in the Riyadh Region between 1986 and 1997 for both rifampicin and streptomycin ${ }^{25}$. The likely culprits are poor compliance and the wide use of rifampin and streptomycin for the treatment of brucellosis and other conditions.

Alternative drug combinations have been used, including other aminoglycosides (e.g., gentamicin and netilmicin), trimethoprimsulfamethoxazole combination and quinolones. A recent meta-analysis of the efficacy of various combinations to treat spondylitis advocated treatment for at least three months; however, the superiority of one specific regimen could not be proved ${ }^{24}$, but preliminary results indicate that quinolones may be cost-effective in spondylitis ${ }^{26}$. In this study, patients received various chemotherapy regimens. The duration of therapy was based on the level of organ involvement. No therapeutic failure was observed with any of the regimens, but the relapse rate was $7 \%$. None of the patients who received streptomycin or ciprofloxacin in combination with doxycycline had a relapse. A regimen of doxycycline and ciprofloxacin is significantly more expensive than the traditional regimens. However, when the protracted duration of other treatment regimens, the economic impact of the residual damage and the economic consequences are considered, the combination of doxycycline and ciprofloxacin may prove cost-effective.

\section{CONCLUSION}

The clinical presentations among our patients emphasize the wide and unexpected spectrum of this disease. Timely diagnosis and treatment of patients with brucellosis require clinical awareness of the full spectrum of signs and symptoms associated with brucellosis, as delay in treatment can result in significant morbidity.

\section{REFERENCES}

1. Young EJ. An overview of human brucellosis. Clin Infect Dis 1995; 21: 283-289.

2. Capasso L. Bacteria in two-millennia-old cheese, and related epizoonoses in Roman populations. $J$ Infect 2002; 45: 122-127.

3. Pappas G, Akritidis N, Bosilkovski M, Tsianos E. Brucellosis. N Engl J Med 2005; 352: 23252336.

4. Young EJ, Brucella species. G.L. G.E. R. Principles and Practice of Infectious Diseases 2000 Philadelphia, PA: Churchill Livingstone: $p p$ 2386-2393.

5. Al-Ballaa SR, Al-Aska A, Kambal A, Al-Hedaithy $M A$. Seasonal variation of culture positive brucellosis at a major teaching hospital. Ann Saudi Med 1994; 14: 12-15.

6. Santini $C$, Baiocchi $P$, Berardelli $A$, Venditti $M$, Serra $P$. A case of brain abscess due to Brucella melitensis. Clin Infect Dis 1994; 19: 977-978.

7. Potasman I, Even L, Banai M, Cohen E, Angel D, Jaffe $M$. Brucellosis: an unusual diagnosis for a seronegative patient with abscesses, osteomeylitis and ulcerative colitis. Rev Clin Dis 1991; 13: 1039-1042.

8. Shakir RA, Al-Din ASN, Araj GF, Lulu AR, Mousa $A R$, Saadah MA. Clinical diagnosis of neurobrucellosis. A report on 19 cases. Brain 1987; 110: 213-223.

9. Colmenero JD, Reguera JM, Martos F, SÃ jnchezDe-Mora $D$, Delgado M, Causse M, MartÃnFarf $\tilde{A}_{i} n A$, JuÃ $i r e z$ C. Complications associated with Brucella melitensis infection: a study of 530 cases. Medicine (Baltimore) 1996; 75: 195211.

10. Galanakis E, Bourantas KL, Leveidiotou S, Lapatsanis $P D$. Childhood brucellosis in northwestern Greece. Eur J Pediatr 1996; 155: 1-6.

11. Navarro-Martinez A, Solera J, Corredoira J, Beato JL. MartÃnez-Alfaro E Atĩ̃̊nzar $M$, Ariza J. Epididymoorchitis due to Brucella mellitensis: a retrospective study of 59 patients. Clin Infect Dis 2001; 33: 2017-2022.

12. Khan MY, Mah MW, Memish ZA. Brucellosis in pregnant women. Clin Infect Dis 2001; 32: 1172-1177. 
13. Cervantes F, Bruguera $M$, Carbonell J, Force $L$, Webb S. Liver disease in brucellosis: a clinical and pathological study of 40 cases. Postgrad Med J 1982; 58: 346-350.

14. Ariza J, Pigrau C, Canas C, Marran A; Martanez F; Corredoira JM; Casanova A; Fabregat $J$; Pahissa A. Current understanding and management of chronic hepatosplenic suppurative brucellosis. Clin Infect Dis 2001; 32: 1024-1033.

15. Gencer $S$, Ozer S, Spontaneous bacterial peritonitis caused by Brucella melitensis. Scand J Infect Dis 2003; 35: 341-343.

16. Taskapan H, Oymak O, Sumerkan B, Tokgoz B, Uta $s C$, Brucella peritonitis in a patient on continuous ambulatory peritoneal dialysis with acute brucellosis. Nephron 2002; 91: 156-158.

17. Memish Z, Mah MW, Al Mahmoud S, Al Shaalan M, Khan MY. Brucella bacteraemia: clinical and laboratory observations in 160 patients. J Infect 2000; 40:59-63.

18. Gotuzzo E, Carrillo C, Guerra J, and Llosa L, An evaluation of diagnostic methods for brucellosis - the value of bone marrow culture. $J$ Infect Dis 1986; 153: 122-125.

19. 17. Solera J, Martínez-Alfaro E, Espinosa $A$. Recognition and optimum treatment of brucellosis. Drug 1997; 53: 245-256.

20. Potasman I, Even L, Banai M, Cohen E, Angel D, Jaffe M. Brucellosis: an unusual diagnosis for a seronegative patient with abscesses, osteomyelitis, and ulcerative colitis. Rev Infect Dis 1991; 13: 1039-1042.

21. Gad El-Rab MO, Kambal IS. Evaluation of a brucella enzyme immunoassay test (ELISA) in comparison with bacteriological culture and agglutination. J Infect 1998; 36: 197-201.

22. Memish ZA, Almuneef $M$, Mah MW , Qassem LA Osoba AO. Comparison of the Brucella Standard Agglutination Test with the ELISA IgG and IgM in patients with Brucella bacteremia. Diagn Microbiol Infect Dis 2002; 44: 129-132.

23. Joint-Food and Agriculture Organization/World Health Organization. FAO/WHO Expert Committee on brucellosis (sixth report) WHO Technical report series No. 740, Geneva, WHO, 1986: pp. 56-57.

24. Solera J, Martinez-Alfaro E, Saez L. Meta-analysis of the efficacy of rifampicin and doxycycline in the treatment of human brucellosis. Med Clin Barc1994; 102: 731-738.

25. AL-Hajjaj S, Kassimi F, Al-Mobeireek AF; Alzeer AH.Progressive rise in mycobacterium tuberculosis resistance to rifampicin and streptomycin in Riyadh, Saudi Arabia: Respirology 2001; 6: 317-322.

26. Pappas G, Seitaridis S, Akritidis N, Tsianos E. Treatment of Brucella spondylitis: lessons from an impossible meta-analysis and initial report of efficacy of a fluoroquinolone-containing regimen. Int J Antimicrob Agents 2004; 24: 502507. 